\title{
Health effects of exposure to second- and third-hand marijuana smoke: a systematic review
}

\author{
Hannah Holitzki BHSc, Laura E. Dowsett MSc, Eldon Spackman PhD, Tom Noseworthy MD MPH, \\ Fiona Clement PhD
}

\section{Abstract}

Background: Recreational marijuana has been legalized in 11 jurisdictions; Canada will legalize marijuana by July 2018 . With this changing landscape, there is a need to understand the public health risks associated with marijuana to support patient-care provider conversations, harm-reduction measures and evidence-informed policy. The objective of this work was to summarize the health effects of exposure to second- and third-hand marijuana smoke.

Methods: In this systematic review, we searched 6 databases from inception to October 2017. Abstract and full-text review was conducted in duplicate. Studies were included if they were human, in vivo or in vitro studies with more than 1 case reported in English or French, and reported original, quantitative data. Three outcomes were extracted: 1) cannabinoids and cannabinoid metabolites in bodily fluids, 2) self-reported psychoactive effects and 3) eye irritation and discomfort.

Results: Of the 1701 abstracts identified, 60 proceeded to full-text review; the final data set contained 15 articles. All of the included studies were of good to poor quality as assessed with the Downs and Black checklist. There is evidence of a direct relation between the tetrahydrocannabinol content of marijuana and effects on those passively exposed. This relation is mediated by several environmental factors including the amount of smoke, ventilation, air volume, number of marijuana cigarettes lit and number of smokers present. No evidence was identified assessing exposure to third-hand marijuana smoke or the health effects of long-term exposure.

Interpretation: Exposure to second-hand marijuana smoke leads to cannabinoid metabolites in bodily fluids, and people experience psychoactive effects after such exposure. Alignment of tobacco and marijuana smoking bylaws may result in the most effective public policies. More research is required to understand the impact of exposure to third-hand smoke and the health effects of long-term exposure to second-hand smoke.

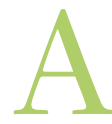

2012 nationally representative Canadian study showed that $12.2 \%$ of people aged 15 years or more had used marijuana in the previous 12 months, and $42.5 \%$ reported marijuana use at least once in their lifetime. ${ }^{1}$ Harms associated with direct marijuana use from the literature include a higher risk of mental illness, a higher risk of being involved in a motor vehicle crash and negative effects on brain development in adolescents. ${ }^{2}$ However, the effects of passive exposure to marijuana smoke remain largely unknown. Effects of passive exposure to tobacco smoke, including both second- and third-hand smoke, have been reported. ${ }^{3-7}$ Exposure to second-hand smoke ("smoke exhaled by a smoker or is emitted from the burning cigarette that is then inhaled by an individual in close proximity" ${ }^{8}$ ) from tobacco is known to cause fetal anomalies, reproductive complications, respiratory disease, cancers and cardiovascular dis- ease. ${ }^{3-5,9}$ The potential effects of third-hand smoke ("residual tobacco smoke pollution that occurs after smoking") are now also being reported and include DNA damage from exposure to nongaseous particles that react with nitrous acid in the environment. ${ }^{10-14}$ Investigation into the health harms from exposure to second- and third-hand marijuana smoke is limited, but there is preliminary evidence from an animal model that endothelial function is impaired. ${ }^{15}$

\section{Competing interests: None declared.}

This article has been peer reviewed.

Correspondence to: Fiona Clement, fclement@ucalgary.ca

CMAJ Open 2017. DOI:10.9778/cmajo.20170112 
In jurisdictions where marijuana remains an illegal substance, it is difficult to impose regulations or health warnings to try to limit exposure to second- and third-hand smoke, which raises concerns for public health. In the last 5 years, recreational marijuana has been legalized in 6 jurisdictions: Uruguay, Alaska, Colorado, Oregon, Washington and Washington, DC. In November 2016, 5 additional US states voted on proposals to legalize marijuana; the proposals passed in Nevada, California, Maine and Massachusetts, and failed in Arizona. Canada plans to legalize marijuana in 2018, and it is likely that more US states and jurisdictions will legalize the drug in the coming years. With this changing landscape, there is a need to better understand the public and individual health risks associated with exposure to second- and third-hand marijuana smoke. The objective of this work was to synthesize the available evidence on this topic. This information will be important to support evidence-informed policy and to support patient-care provider conversations to reduce harm.

\section{Methods}

\section{Data sources}

We conducted a systematic review of published literature on the effects of exposure to second- and third-hand marijuana smoke, searching 6 databases (MEDLINE, the Cochrane Database of Systematic Reviews, Embase, PsychINFO, CINAHL and the HTA database) from their inception to Oct. 17, 2017. A library and information specialist developed the search strategies. We conducted the search using all $\mathrm{MeSH}$ terms referring to marijuana (e.g., ganga, bhang, hashish, pot, shatter, weed) and $\mathrm{MeSH}$ terms referring to second- or third-hand marijuana smoke (e.g., exposure, involuntary, passive, second-hand, third-hand). The full MEDLINE search strategy is available in Appendix 1 (available at www.cmajopen.ca/content/5/4/E814/suppl/DC1). We followed the PRISMA guidelines ${ }^{16}$ throughout data acquisition and reporting.

\section{Study selection}

Abstract review was conducted independently by 2 reviewers (H.H., L.E.D.). To be included, studies had to be human, in vivo or in vitro studies with more than 1 case reported in English or French, and report original, quantitative data. Abstracts were excluded if they failed to meet all the inclusion criteria; thus, all case reports, commentaries, editorials and letters were excluded. Studies included by either reviewer proceeded to full-text review, which was also conducted by 2 independent reviewers (H.H., L.E.D.). Any disagreements between reviewers were resolved through discussion of the full text; if required, a third reviewer (F.C.) was consulted. After full-text review, the reference lists of included studies were searched to ensure no relevant studies were overlooked.

\section{Data extraction}

Data extraction was performed by 2 reviewers (H.H., L.E.D.) in 2017 and included details on the design of the intervention, number of experimental trials, length of exposure, participant recruitment methods, number of participants, inclusion criteria and participant characteristics. Three outcomes were extracted: 1) cannabinoids and cannabinoid metabolites in bodily fluids, 2) self-reported psychoactive effects and 3) eye irritation and discomfort.

\section{Quality assessment}

We assessed the quality of the included studies in duplicate using the Downs and Black checklist, a 27-item checklist for assessing the methodological quality of both randomized and nonrandomized studies of health care interventions. ${ }^{17}$ The checklist has 5 constructs: 1) reporting, 2) external validity, 3) internal validity — bias, 4) internal validity - confounding and 5) power. ${ }^{17}$ Studies are assigned a score of 1 or 0 for each criterion, with a higher score indicating higher quality; the maximum score is 28 . Each paper was assigned a grade of excellent (24-28 points), good (19-23 points), fair (1418 points) or poor (<14 points). ${ }^{18}$

\section{Analysis}

We categorized records as studies that measured the chemical components of marijuana smoke or those that investigated the immediate effects on people exposed to second- or third-hand marijuana smoke. Based on outcomes reported, the studies that investigated the immediate effects of exposure were further categorized into 3 subcategories: cannabinoids (e.g., tetrahydrocannabinol [THC]) and metabolites in bodily fluids, impact of ventilation on effects of second- or third-hand smoke exposure and psychoactive effects of passive exposure. We synthesized the findings within each category qualitatively. Synthesis involved reporting aspects of the findings that were similar or, if there were discrepancies between studies, reporting the differences in study design, methods or execution that could account for the differences.

\section{Results}

We identified 1701 unique abstracts. Of these, 60 proceeded to full-text review, 15 of which were included in the final data set (Figure 1). The 15 records reported findings from 8 unique studies. ${ }^{19-33}$ Details of each included study are presented in Table 1.

All 15 records were experimental studies on the immediate effects of marijuana smoke exposure in humans in a controlled environment. They all followed a similar protocol whereby nonsmokers sat in proximity to people who were actively smoking. Physiological or psychological outcomes were measured after a period of exposure. ${ }^{19-30,32,33}$ None of the included studies investigated third-hand marijuana smoke.

All included studies assessed short-term (within $24 \mathrm{~h}$ of exposure) effects of smoke exposure; none assessed health effects beyond 24 hours. Meta-analysis was not possible owing to heterogeneous outcomes and reporting, and, therefore, the included studies were synthesized narratively.

All of the included studies were of good to poor quality. The average score on quality assessment was 17.8 , with a 
range of $13^{27}$ to $22^{22-25}$ (the latter being experimental designs with multiple trials completed).

\section{Immediate clinical outcomes from marijuana smoke exposure}

\section{Cannabinoids and cannabinoid metabolites in bodily fluids} Oral fluid concentrations of THC were reported in 4 reports from 3 studies. ${ }^{20-22,28}$ All studies showed THC in the oral fluid of participants who had been exposed to second-hand marijuana smoke.

Blood concentrations of THC were measured in 8 reports from 6 studies. ${ }^{22,24-27,30,31,33}$ Participants exposed to secondhand marijuana smoke had lower blood concentrations of THC than active smokers; ${ }^{22,24,27,33}$ however, there were detectable amounts of THC in the blood samples of those exposed to second-hand smoke..$^{25,26,30,31}$ In 1 study in which multiple trials were performed to test marijuana of different THC content $(5.3 \%$ and $11.3 \%)$, there were no significant differences in the blood concentrations of THC and THC metabolites between trial groups. ${ }^{22}$

In 13 reports from 9 studies, the investigators assessed THC metabolite concentrations in urine samples. ${ }^{19-21,23-27,29-33}$ The evidence suggests that a higher percentage of THC content in smoked marijuana results in higher THC metabolite content in urine (Figure 2). ${ }^{20,22,27}$ For example, 4 hours after exposure to marijuana with $1.5 \%$ THC, 1 of 5 participants had more than the $20 \mathrm{ng} / \mathrm{mL}$ threshold for urine testing, and 4 hours after exposure to marijuana with $11.3 \%$ THC, all participants had more than $15 \mathrm{ng} / \mathrm{mL}$, with a maximum concentration of cannabinoid metabolites of $28.3 \mathrm{ng} / \mathrm{mL}$ in the urine..$^{23,33}$

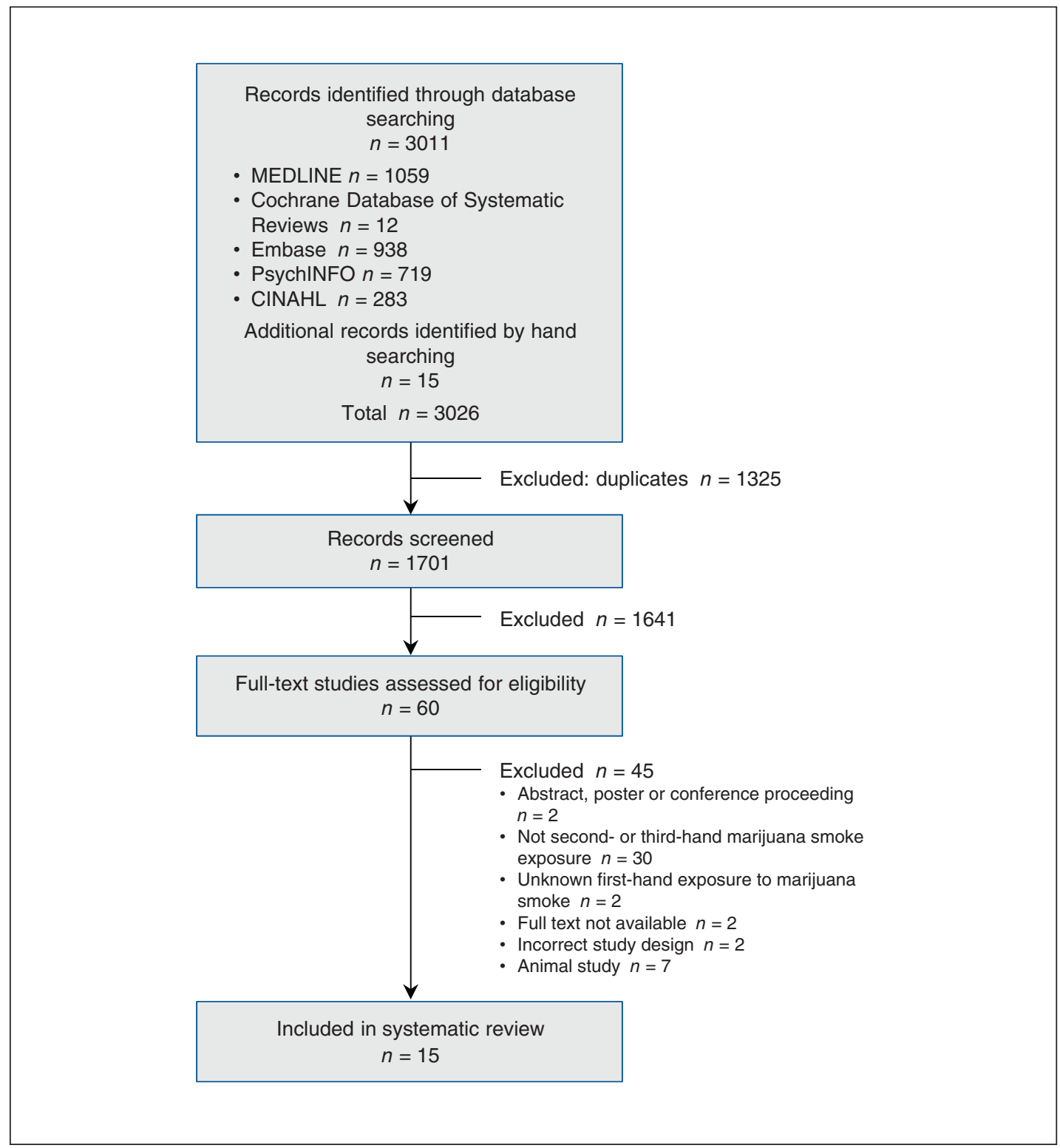

Figure 1: Flow chart of identified records. 
In 2 studies, multiple trials were conducted in ventilated and unventilated environments. ${ }^{19,22-24}$ Ventilation was manipulated by opening a door ${ }^{19}$ or altering the air circulation rate in the room. ${ }^{22-24}$ Both urine THC metabolite concentrations and blood THC levels were higher in those exposed to secondhand smoke in an unventilated environment than in a ventilated environment. Other factors that mediated the effects of exposure to second-hand smoke included air volume, number

\begin{tabular}{|c|c|c|c|c|c|c|}
\hline $\begin{array}{l}\text { Author/ } \\
\text { country }\end{array}$ & Intervention & Participant selection & $\begin{array}{c}\text { No. of } \\
\text { participants }\end{array}$ & $\begin{array}{c}\text { Participant } \\
\text { characteristics }\end{array}$ & Reported outcomes & Quality* \\
\hline \multirow{2}{*}{$\begin{array}{l}\text { Cone et al.,22 } \\
2015, \text { United } \\
\text { States }\end{array}$} & \multirow[b]{2}{*}{$\begin{array}{l}\text { Intervention: nonsmokers were } \\
\text { exposed to marijuana smoke } \\
\text { from participants smoking } \\
\text { marijuana in controlled } \\
\text { environment laboratory over } \\
3 \text { sessions; potency and } \\
\text { ventilation of environment were } \\
\text { changed between each session } \\
\text { Multiple trials: 1) } 5.3 \% \text { THC in } \\
\text { unventilated environment, } \\
\text { 2) } 11.3 \% \text { THC in unventilated } \\
\text { environment, 3) } 11.3 \% \text { THC in } \\
\text { ventilated environment }\end{array}$} & \multirow[b]{2}{*}{$\begin{array}{l}\text { Participant selection: recruited } \\
\text { through newspaper } \\
\text { advertisements, flyers posted on } \\
\text { university campus and around } \\
\text { community, and word of mouth } \\
\text { Inclusion criteria for smokers: } \\
\text { self-reported use of cannabis at } \\
\text { least } 2 \text { times per week during } \\
\text { previous } 90 \mathrm{~d} \text {, negative results of } \\
\text { testing for other illicit substances } \\
\text { Inclusion criteria for nonsmokers: } \\
\text { healthy participants who } \\
\text { self-reported lifetime cannabis } \\
\text { use but had not used cannabis } \\
\text { or any other illicit drug in } \\
\text { previous } 6 \text { mo }\end{array}$} & 6 smokers & NR & \multirow{2}{*}{$\begin{array}{l}\text { THC level in oral fluid } \\
\text { and whole blood, } \\
\text { self-report of drug effects } \\
\text { (Drug Effects } \\
\text { Questionnaire visual } \\
\text { analogue scale) }\end{array}$} & \multirow[t]{2}{*}{22} \\
\hline & & & 6 nonsmokers & NR & & \\
\hline \multirow[t]{2}{*}{$\begin{array}{l}\text { Cone et al.., }{ }^{23} \\
\text { 2015, United } \\
\text { States }\end{array}$} & \multirow{2}{*}{$\begin{array}{l}\text { Intervention: nonsmokers were } \\
\text { exposed to marijuana smoke } \\
\text { from participants smoking } \\
\text { marijuana in controlled } \\
\text { environment laboratory over } \\
3 \text { sessions; potency and } \\
\text { ventilation of environment were } \\
\text { changed between each session } \\
\text { Multiple trials: 1) } 5.3 \% \text { THC in } \\
\text { unventilated environment, } \\
2 \text { ) } 11.3 \% \text { THC in unventilated } \\
\text { environment, 3) } 11.3 \% \text { THC in } \\
\text { ventilated environment }\end{array}$} & \multirow{2}{*}{$\begin{array}{l}\text { Participant selection: recruited } \\
\text { through newspaper } \\
\text { advertisements, flyers posted on } \\
\text { university campus and around } \\
\text { community, and word of mouth } \\
\text { Inclusion criteria for smokers: } \\
\text { self-reported use of cannabis at } \\
\text { least } 2 \text { times per week during } \\
\text { previous } 90 \mathrm{~d} \text {, negative results of } \\
\text { testing for other illicit substances } \\
\text { Inclusion criteria for nonsmokers: } \\
\text { healthy participants who } \\
\text { self-reported lifetime cannabis } \\
\text { use but had not used cannabis } \\
\text { or any other illicit drug in } \\
\text { previous } 6 \text { mo }\end{array}$} & 8 smokers & $\begin{array}{l}3 \text { women, } \\
5 \text { men, average } \\
\text { age } 29 \text { (SD } \\
6 \text { ) yr, average } \\
\text { BMl } 25.6\end{array}$ & \multirow[t]{2}{*}{$\begin{array}{l}\text { Total cannabis use } \\
\text { (weight), THC level in } \\
\text { urine }\end{array}$} & \multirow[t]{2}{*}{22} \\
\hline & & & 18 nonsmokers & $\begin{array}{l}9 \text { women, } \\
9 \text { men, average } \\
\text { age } 28 \text { (SD 7) } \\
\text { yr, average } \\
\text { BMl } 24.7\end{array}$ & & \\
\hline $\begin{array}{l}\text { Cone et al., }{ }^{19} \\
\text { 1987, United } \\
\text { States }\end{array}$ & $\begin{array}{l}\text { Intervention: nonsmokers were } \\
\text { exposed to marijuana cigarette } \\
\text { smoke }(2.8 \% \text { THC) under } \\
\text { double-blind conditions } \\
\text { Multiple trials: } 3 \text { trials, } 1 \text { with } \\
4 \text { cigarettes and } 2 \text { with } \\
16 \text { cigarettes }\end{array}$ & $\begin{array}{l}\text { Participant selection: NR } \\
\text { Inclusion criteria for nonsmokers: } \\
\text { healthy, drug-free men with } \\
\text { history of marijuana use who } \\
\text { had } 14 \text { consecutive d of } \\
\text { cannabinoid-free urine tests; } \\
2 \text { cannabis-naive men (members } \\
\text { of research team) }\end{array}$ & 7 nonsmokers & $\begin{array}{l}\text { All men, } \\
\text { average age } \\
36 \mathrm{yr} \text {, average } \\
\text { weight } 74.7 \mathrm{~kg}\end{array}$ & $\begin{array}{l}\text { Room air THC } \\
\text { concentrations, THC } \\
\text { level in urine }\end{array}$ & 20 \\
\hline $\begin{array}{l}\text { Cone et al., }{ }^{25} \\
\text { 1986, United } \\
\text { States }\end{array}$ & $\begin{array}{l}\text { Intervention: nonsmokers were } \\
\text { exposed to marijuana cigarette } \\
\text { smoke }(2.8 \% \text { THC) under } \\
\text { double-blind conditions } \\
\text { Multiple trials: } 3 \text { trials, } 1 \text { with } \\
4 \text { cigarettes and } 2 \text { with } \\
16 \text { cigarettes }\end{array}$ & $\begin{array}{l}\text { Participant selection: NR } \\
\text { Inclusion criteria for nonsmokers: } \\
\text { healthy, drug-free men with } \\
\text { history of marijuana use who } \\
\text { had } 14 \text { consecutive d of } \\
\text { cannabinoid-free urine tests; } \\
2 \text { cannabis-naive men }\end{array}$ & 7 nonsmokers & $\begin{array}{l}\text { All men, } \\
\text { average age } \\
36 \mathrm{yr} \text {, average } \\
\text { weight } 74.7 \mathrm{~kg}\end{array}$ & $\begin{array}{l}\text { THC level in urine (EMIT } \\
20 \mathrm{ng} / \mathrm{mL} \text { and } 100 \mathrm{ng} / \\
\mathrm{mL} \text { ) and whole blood, } \\
\text { heart rate, blood } \\
\text { pressure, subscales of } \\
\text { Addiction Research } \\
\text { Center Inventory } \\
\text { (single-dose } \\
\text { questionnaire, visual } \\
\text { analogue scale) }\end{array}$ & 22 \\
\hline $\begin{array}{l}\text { Cone et al., }{ }^{26} \\
\text { 1986, United } \\
\text { States }\end{array}$ & $\begin{array}{l}\text { Intervention: nonsmokers were } \\
\text { exposed to marijuana cigarette } \\
\text { smoke }(2.8 \% \text { THC) under } \\
\text { double-blind conditions } \\
\text { Multiple trials: } 3 \text { trials, } 1 \text { with } \\
4 \text { cigarettes and } 2 \text { with } \\
16 \text { cigarettes }\end{array}$ & $\begin{array}{l}\text { Participant selection: NR } \\
\text { Inclusion criteria for nonsmokers: } \\
\text { healthy, drug-free men with } \\
\text { history of marijuana use who } \\
\text { had } 14 \text { consecutive } d \text { of } \\
\text { cannabinoid-free urine tests; } \\
2 \text { cannabis-naive men }\end{array}$ & 7 nonsmokers & $\begin{array}{l}\text { All men, } \\
\text { average age } \\
36 \mathrm{yr} \text {, average } \\
\text { weight } 74.7 \mathrm{~kg}\end{array}$ & $\begin{array}{l}\text { THC level in urine (EMIT } \\
20 \mathrm{ng} / \mathrm{mL} \text { and } 100 \mathrm{ng} / \\
\mathrm{mL} \text { ) and whole blood, } \\
\text { subscales of Addiction } \\
\text { Research Center } \\
\text { Inventory (single-dose } \\
\text { questionnaire, visual } \\
\text { analogue scale, circular } \\
\text { lights task, digit-symbol } \\
\text { substitution task) }\end{array}$ & 19 \\
\hline
\end{tabular}




\section{Table 1 (part 2 of 3): Characteristics of included studies}

\begin{tabular}{|c|c|c|c|c|c|c|}
\hline $\begin{array}{l}\text { Author/ } \\
\text { country }\end{array}$ & Intervention & Participant selection & $\begin{array}{c}\text { No. of } \\
\text { participants }\end{array}$ & $\begin{array}{c}\text { Participant } \\
\text { characteristics }\end{array}$ & Reported outcomes & Quality* \\
\hline \multirow[t]{2}{*}{$\begin{array}{l}\text { Herrmann et } \\
\text { al. }{ }^{24} 2015, \\
\text { United States }\end{array}$} & \multirow{2}{*}{$\begin{array}{l}\text { Intervention: nonsmokers were } \\
\text { exposed to marijuana smoke } \\
\text { from participants smoking } \\
\text { marijuana in controlled } \\
\text { environment laboratory over } \\
3 \text { sessions; unlimited marijuana } \\
\text { was provided to smokers } \\
\text { Multiple trials: } 1 \text { ) } 11.3 \% \text { THC in } \\
\text { unventilated environment, } \\
2 \text { ) } 11.3 \% \text { THC in ventilated } \\
\text { environment (11 air exchanges } \\
\text { per hour) }\end{array}$} & \multirow{2}{*}{$\begin{array}{l}\text { Participant selection: recruited } \\
\text { from Baltimore through media } \\
\text { advertising and word of mouth } \\
\text { Inclusion criteria for smokers: } \\
\text { age } 18-45 \mathrm{yr} \text {, used cannabis at } \\
\text { least } 2 \text { times per week during } \\
\text { previous } 90 \mathrm{~d} \text {, urine sample } \\
\text { positive for THC and negative for } \\
\text { other drugs, negative breath } \\
\text { alcohol reading at screening and } \\
\text { on day of session, BMl 19-34, } \\
\text { not pregnant or nursing } \\
\text { Inclusion criteria for nonsmokers: } \\
\text { age } 18-45 \text { yr, cannabis use at } \\
\text { least once but not during } \\
\text { previous } 6 \text { mo, urine sample } \\
\text { negative for all drugs, negative } \\
\text { breath alcohol reading at } \\
\text { screening and on day of session, } \\
\text { BMl } 19-34 \text {, not pregnant or } \\
\text { nursing }\end{array}$} & 7 smokers & $\begin{array}{l}4 \text { men, } \\
3 \text { women, } \\
\text { average age } \\
29.4 \text { (SD } \\
5.8 \text { ) yr, average } \\
\text { BMl } 25.6\end{array}$ & \multirow{2}{*}{$\begin{array}{l}\text { Total weight of cannabis } \\
\text { smoked, THC level in } \\
\text { blood and urine, heart } \\
\text { rate, blood pressure, } \\
\text { subscales of Drug } \\
\text { Effects Questionnaire } \\
\text { (divided attention task, } \\
\text { digit-symbol substitution } \\
\text { task, paced auditory } \\
\text { serial addition task) }\end{array}$} & \multirow[t]{2}{*}{22} \\
\hline & & & 12 nonsmokers & $\begin{array}{l}3 \text { men, } \\
3 \text { women, } \\
\text { average age } \\
28.7 \mathrm{yr}, \\
\text { average BMI } \\
25.3\end{array}$ & & \\
\hline \multirow{2}{*}{$\begin{array}{l}\text { Law et al., }{ }^{27} \\
\text { 1984, United } \\
\text { Kingdom }\end{array}$} & \multirow[b]{2}{*}{$\begin{array}{l}\text { Intervention: nonsmokers were } \\
\text { exposed to marijuana smoke } \\
\text { (9.8\% THC) in a small, } \\
\text { unventilated room } \\
\text { Multiple trials: No } \\
\text { Timeline of exposure: after } \\
\text { smokers had consumed their } \\
\text { cannabis cigarette (which took } \\
10-34 \text { min), nonsmoking } \\
\text { participants remained in room for } \\
3 \mathrm{~h}\end{array}$} & \multirow{2}{*}{$\begin{array}{l}\text { Participant selection: NR } \\
\text { Inclusion criteria for smokers: NR } \\
\text { Inclusion criteria for nonsmokers: } \\
\text { NR }\end{array}$} & 6 smokers & NR & \multirow{2}{*}{$\begin{array}{l}\text { Environmental exposure } \\
\text { (gas chromatography), } \\
\text { THC level in urine and } \\
\text { whole blood } \\
\text { (radioimmunoassay) }\end{array}$} & \multirow[t]{2}{*}{13} \\
\hline & & & 4 nonsmokers & NR & & \\
\hline \multirow[t]{2}{*}{$\begin{array}{l}\text { Moore et } \\
\text { al. }{ }^{28} 2011 \\
\text { United States }\end{array}$} & \multirow{2}{*}{$\begin{array}{l}\text { Intervention: passive } 3-\mathrm{h} \\
\text { exposure to marijuana in Dutch } \\
\text { "coffee shop" } \\
\text { Multiple trials: } 2 \text { trials in } \\
2 \text { different coffee shops, with } \\
\text { varying numbers of active } \\
\text { smokers (varying THC } \\
\text { percentage) }\end{array}$} & \multirow{2}{*}{$\begin{array}{l}\text { Participant selection: volunteers; } \\
\text { selection strategy NR } \\
\text { Inclusion criteria for smokers: } \\
\text { any active smoker in coffee shop } \\
\text { during exposure timeline } \\
\text { Inclusion criteria for nonsmokers: } \\
\text { healthy participants who did not } \\
\text { smoke marijuana }\end{array}$} & $\begin{array}{l}16 \text { smokers in } \\
\text { trial } 1 \text {, } \\
6 \text { smokers in } \\
\text { trial } 2\end{array}$ & NR & \multirow{2}{*}{$\begin{array}{l}\text { Air cannabinoid content } \\
\text { (Quantisal collection } \\
\text { device), THC level in oral } \\
\text { fluid (Quantisal collection } \\
\text { device) }\end{array}$} & \multirow[t]{2}{*}{19} \\
\hline & & & 10 nonsmokers & $\begin{array}{l}5 \text { men, average } \\
\text { age } 22.8 \mathrm{yr}, \\
\text { average weight } \\
84 \mathrm{~kg} \text {, average } \\
\text { height } 1.9 \mathrm{~m} \text {, } \\
\text { average BMl } \\
233 ; 5 \text { women, } \\
\text { average age } \\
23.8 \mathrm{yr} \text {, } \\
\text { average weight } \\
62.4 \mathrm{~kg}, \\
\text { average height } \\
1.71 \mathrm{~m}, \\
\text { average BMl } \\
21.2\end{array}$ & & \\
\hline \multirow{2}{*}{$\begin{array}{l}\text { Mørland et } \\
\text { al., }{ }^{33} 1985 \text {, } \\
\text { Norway }\end{array}$} & \multirow{2}{*}{$\begin{array}{l}\text { Intervention: participants were } \\
\text { exposed to marijuana and } \\
\text { hashish smoke in small, } \\
\text { unventilated car } \\
\text { Multiple trials: } 1 \text { ) hashish (1.5\% } \\
\text { THC), 2) marijuana (1.5\% THC) }\end{array}$} & \multirow{2}{*}{$\begin{array}{l}\text { Participant selection: volunteers; } \\
\text { selection strategy NR } \\
\text { Inclusion criteria for smokers: NR } \\
\text { Inclusion criteria for nonsmokers: } \\
\text { healthy cannabis-naive } \\
\text { participants }\end{array}$} & 5 smokers & NR & \multirow{2}{*}{$\begin{array}{l}\text { Blood cannabinoid levels } \\
\text { (radioimmunoassay), } \\
\text { THC level in urine (EMIT) }\end{array}$} & \multirow[t]{2}{*}{16} \\
\hline & & & 10 nonsmokers & $\begin{array}{l}7 \text { men, } \\
3 \text { women "of } \\
\text { normal weight } \\
\text { in relation to } \\
\text { their height, } \\
\text { age, and sex" }\end{array}$ & & \\
\hline \multirow[t]{2}{*}{$\begin{array}{l}\text { Mulé et al. }{ }^{29} \\
1988, \text { United } \\
\text { States }\end{array}$} & \multirow[t]{2}{*}{$\begin{array}{l}\text { Intervention: in first part of } \\
\text { experiment, smokers were asked } \\
\text { to smoke cannabis as they } \\
\text { usually did and were observed; in } \\
\text { second part, nonsmokers were } \\
\text { exposed to smoke of } 4 \text { cannabis } \\
\text { cigarettes ( } 27 \text { mg THC) in } \\
\text { unventilated room } \\
\text { Multiple trials: no }\end{array}$} & \multirow[t]{2}{*}{$\begin{array}{l}\text { Participant selection: NR } \\
\text { Inclusion criteria for smokers: } \\
\text { occasional ( } 1 \text { cigarette/wk) or } \\
\text { moderate ( } 1-3 \text { cigarettes/wk) } \\
\text { smoking } \\
\text { Inclusion criteria for nonsmokers: } \\
\text { NR }\end{array}$} & 8 smokers & $\begin{array}{l}\text { All male, age } \\
21-27 \mathrm{yr}, \\
\text { height 5'9"-6' } 1 " \\
(1.75-1.85 \mathrm{~m}), \\
\text { weight } \\
154-175 \mathrm{lbs} \\
(69.8-79.4 \mathrm{~kg})\end{array}$ & \multirow[t]{2}{*}{ THC level in urine (EMIT) } & \multirow[t]{2}{*}{18} \\
\hline & & & 3 nonsmokers & $\mathrm{NR}$ & & \\
\hline
\end{tabular}




\section{Table 1 (part 3 of 3): Characteristics of included studies}

\begin{tabular}{|c|c|c|c|c|c|c|}
\hline $\begin{array}{l}\text { Author/ } \\
\text { country }\end{array}$ & Intervention & Participant selection & $\begin{array}{c}\text { No. of } \\
\text { participants }\end{array}$ & $\begin{array}{c}\text { Participant } \\
\text { characteristics }\end{array}$ & Reported outcomes & Quality* \\
\hline \multirow{2}{*}{$\begin{array}{l}\text { Niedbala et } \\
\text { al., }{ }^{20} 2005, \\
\text { United States }\end{array}$} & \multirow{2}{*}{$\begin{array}{l}\text { Intervention: participants were } \\
\text { placed in severe second-hand } \\
\text { smoke conditions in unventilated } \\
\text { van for } 1 \mathrm{~h} \\
\text { Multiple trials: } 2 \text { trials, each with } \\
4 \text { smokers and } 4 \text { passive } \\
\text { inhalers; } 5.4 \% \text { THC in trial } 1 \text {, } \\
10.4 \% \text { THC in trial } 2\end{array}$} & \multirow{2}{*}{$\begin{array}{l}\text { Participant selection: volunteers; } \\
\text { recruitment strategy NR } \\
\text { Inclusion criteria for smokers: } \\
\text { healthy white men who reported } \\
\text { infrequent past cannabis use } \\
\text { Inclusion criteria for } \\
\text { nonsmokers: healthy white men } \\
\text { who tested as cannabis-free } \\
\text { before study based on oral fluid } \\
\text { and urine tests and self-reported } \\
\text { data }\end{array}$} & 8 smokers & $\begin{array}{l}18-24 \text { yr for } \\
\text { both groups }\end{array}$ & \multirow{2}{*}{$\begin{array}{l}\text { THC level in oral fluid } \\
\text { (Intercept collector pads) } \\
\text { and urine }\end{array}$} & \multirow[t]{2}{*}{16} \\
\hline & & & 8 nonsmokers & $\begin{array}{l}34-50 \text { yr in first } \\
\text { group, } \\
25-50 \text { yr in } \\
\text { second group }\end{array}$ & & \\
\hline \multirow{2}{*}{$\begin{array}{l}\text { Niedbala et } \\
\text { al., }{ }^{21} 2004, \\
\text { United States }\end{array}$} & \multirow{2}{*}{$\begin{array}{l}\text { Intervention: smokers consumed } \\
1 \text { cannabis cigarette each } \\
\text { (approximate THC level 1.75\%) in } \\
\text { presence of nonsmokers in } \\
\text { sealed room } \\
\text { Multiple trials: no }\end{array}$} & \multirow[b]{2}{*}{$\begin{array}{l}\text { Participant selection: volunteers; } \\
\text { recruitment strategy NR } \\
\text { Inclusion criteria for smokers: } \\
\text { healthy white men who reported } \\
\text { infrequent prior use of cannabis } \\
\text { Inclusion criteria for } \\
\text { nonsmokers: healthy white men } \\
\text { who tested as cannabis-free } \\
\text { before start of study }\end{array}$} & 5 smokers & Age 21-25 yr & \multirow{2}{*}{$\begin{array}{l}\text { Air cannabinoid content, } \\
\text { THC level in oral fluid } \\
\text { and urine }\end{array}$} & \multirow[t]{2}{*}{15} \\
\hline & & & 4 nonsmokers & Age $37-49 \mathrm{yr}$ & & \\
\hline \multirow[t]{2}{*}{$\begin{array}{l}\text { Perez-Reyes } \\
\text { et al., }{ }^{30} 1983, \\
\text { United States }\end{array}$} & \multirow{2}{*}{$\begin{array}{l}\text { Intervention: smokers consumed } \\
\text { cannabis cigarettes in presence } \\
\text { of nonsmokers in a room (trials } 1 \\
\text { and } 3 \text { ) and a car (trial } 2 \text { ); } \\
\text { biological samples were then } \\
\text { taken and compared between } \\
\text { the } 2 \text { groups } \\
\text { Multiple trials: } 1 \text { ) } 2 \text { cigarettes } \\
\text { (2.5\% and } 2.8 \% \text { THC), } \\
\text { 2) } 2 \text { cigarettes ( } 2.8 \% \text { THC), } \\
\text { 3) } 4 \text { cigarettes }(2.8 \% \text { THC) }\end{array}$} & \multirow[t]{2}{*}{$\begin{array}{l}\text { Participant selection: NR } \\
\text { Inclusion criteria for smokers: } \\
\text { experienced marijuana users } \\
\text { Inclusion criteria for } \\
\text { nonsmokers: marijuana-naive } \\
\text { participants }\end{array}$} & 6 smokers & $\begin{array}{l}3 \text { men, } 3 \\
\text { women, } \\
\text { "healthy and of } \\
\text { normal weight } \\
\text { and height in } \\
\text { relation to their } \\
\text { age and sex" }\end{array}$ & \multirow[t]{2}{*}{$\begin{array}{l}\text { THC presence in air, } \\
\text { THC level in urine (EMIT) } \\
\text { and blood }\end{array}$} & \multirow[t]{2}{*}{16} \\
\hline & & & 6 nonsmokers & $\begin{array}{l}3 \text { men, } 3 \\
\text { women, } \\
\text { "healthy and of } \\
\text { normal weight } \\
\text { and height in } \\
\text { relation to their } \\
\text { age and sex" }\end{array}$ & & \\
\hline \multirow{2}{*}{$\begin{array}{l}\text { Röhrich et } \\
\text { al., }{ }^{31} 2010 \text {, } \\
\text { Germany }\end{array}$} & \multirow{2}{*}{$\begin{array}{l}\text { Intervention: nonsmokers were } \\
\text { exposed to marijuana smoke in } \\
\text { Dutch coffee shop with ventilation } \\
\text { (THC percentage NR) } \\
\text { Multiple trials: no }\end{array}$} & \multirow{2}{*}{$\begin{array}{l}\text { Participant selection: NR } \\
\text { Inclusion criteria for smokers: } \\
\text { active smoker in coffee shop at } \\
\text { time of experiment } \\
\text { Inclusion criteria for } \\
\text { nonsmokers: no history of } \\
\text { cannabis use, no contact with } \\
\text { cannabis in month preceding } \\
\text { experiment }\end{array}$} & $\begin{array}{l}8-25 \text { smokers } \\
\text { at a time }\end{array}$ & NR & \multirow{2}{*}{$\begin{array}{l}\text { THC level in blood and } \\
\text { urine (gas } \\
\text { chromatography-mass } \\
\text { spectrometry) }\end{array}$} & \multirow[t]{2}{*}{15} \\
\hline & & & 8 nonsmokers & $\begin{array}{l}4 \text { men, } \\
4 \text { women }\end{array}$ & & \\
\hline \multirow{2}{*}{$\begin{array}{l}\text { Zeidenberg } \\
\text { et al., }{ }^{32} 1977, \\
\text { United States }\end{array}$} & \multirow{2}{*}{$\begin{array}{l}\text { Intervention: heavy marijuana } \\
\text { smokers consumed cannabis } \\
\text { (THC level NR) in presence of } \\
\text { placebo smoker in locked ward } \\
\text { Multiple trials: no }\end{array}$} & \multirow{2}{*}{$\begin{array}{l}\text { Participant selection: NR } \\
\text { Inclusion criteria for smokers: NR } \\
\text { Inclusion criteria for nonsmoker: } \\
\text { NR }\end{array}$} & 5 smokers & NR & \multirow{2}{*}{$\begin{array}{l}\text { THC level in urine, } \\
\text { subjective reporting, } \\
\text { physical examination }\end{array}$} & \multirow[t]{2}{*}{14} \\
\hline & & & 1 nonsmoker & NR & & \\
\hline
\end{tabular}

of participants inhaling second-hand smoke, THC content, number of marijuana cigarettes lit and number of active smokers. 22,23

\section{Psychoactive effects}

In 2 studies, the psychoactive effects reported by participants exposed to second-hand smoke were described..$^{19,22,32}$ In 1 study, the investigators used a validated measure (Drug Effects Questionnaire), ${ }^{19}$ and in the other, a self-reported feeling of "high" was used. ${ }^{22,32}$ Those exposed to marijuana with higher THC content reported stronger drug effects (Figure 2).22,32 The same trend was reported in active smokers. ${ }^{22,32}$ These data indicate that active smokers and those exposed to second-hand smoke experience a similar pattern of intoxication; however, the latter consistently report weaker drug effects than active smokers. ${ }^{22}$

\section{Discomfort and eye irritation}

In 1 study, participants exposed to second-hand smoke reported discomfort and eye irritation due to smoke in the room..$^{23}$ During the experiment, all participants expressed 


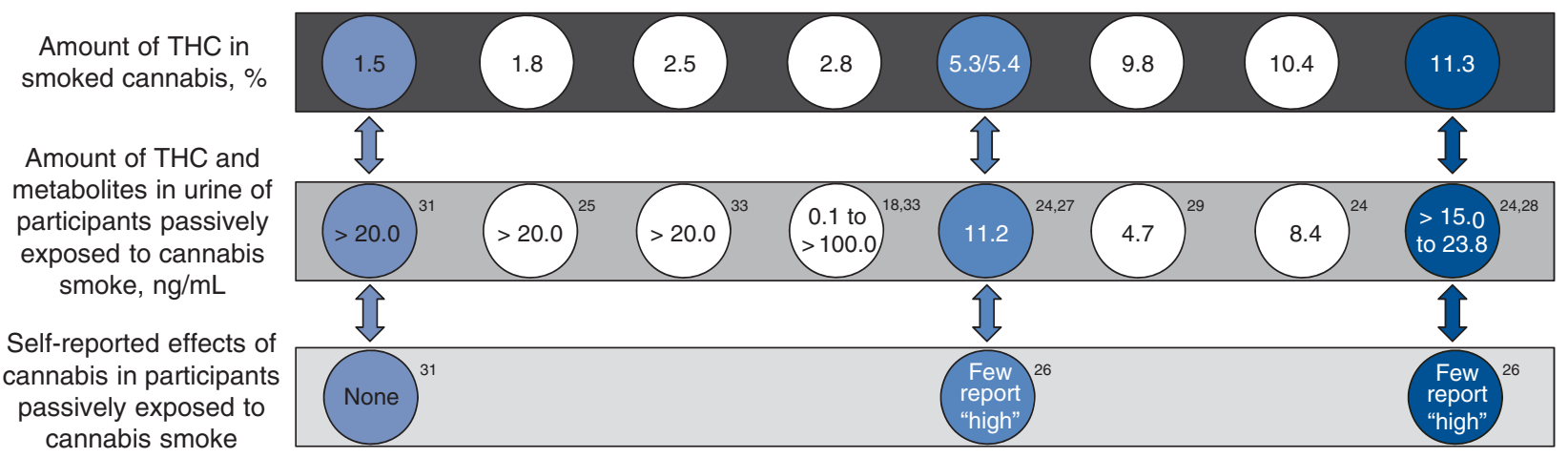

Figure 2: Urine levels of tetrahydrocannabinol (THC) and metabolites and subjective effects in participants passively exposed to marijuana smoke in an unventilated environment, 4-8 hours after exposure, by THC content.

discomfort. ${ }^{23}$ As a result, active smokers ceased smoking when they otherwise would have continued.

\section{Interpretation}

Second-hand exposure to marijuana smoke can lead to cannabinoid metabolites in bodily fluids sufficient for positive results on testing of oral fluids, blood and urine, and can lead to psychoactive effects. There is evidence of a weak doseresponse relation between THC content of cannabis and effects on those exposed to second-hand smoke, including metabolites found in blood and urine, and psychoactive effects. There is evidence that the relation is mediated by environmental factors, including whether the air space is ventilated, volume of air, number of marijuana cigarettes lit at 1 time, potency of the marijuana and number of smokers.

The simulated environments within some of the included studies may not represent "real-world" scenarios. Some studies placed participants in simulated environments where they were exposed to smoke in closed rooms with controlled ventilation systems. In the context of legalization, people may be exposed to second-hand marijuana smoke outside, in parks or in passing on the sidewalk. This type of exposure may not result in cannabinoid metabolites in bodily fluids, as the exposure may be shorter and less intense than in unventilated areas. However, exposure in closed spaces such as in cafés, bars and clubs may occur, depending on the regulations prohibiting smoking in indoor spaces. In addition, exposure in unventilated spaces such as vehicles or small rooms in private homes is still likely to occur. Thus, the observed relation between second-hand smoke exposure and cannabinoid metabolites in bodily fluids is likely to be generalizable to real-world contexts. Marijuana use in enclosed spaces, particularly in the presence of children, older people or people with respiratory illness, should be limited, ideally through public health measures and legislation in jurisdictions where marijuana is legalized.
In some domains, mirroring public health legislation to protect workers and the general public from second-hand tobacco exposure will be appropriate. For example, bylaws forbidding smoking in indoor spaces such as bars and nightclubs and in shared outdoor spaces such as beaches or parks should be considered. Tobacco smoking frameworks may be useful to inform control regulation. Alignment of tobacco and marijuana smoking bylaws, with a coherent policy approach to exposure to smoke of any kind, may result in the most effective public policies.

Evidence suggests that the chemical composition of second-hand marijuana smoke is similar to that of secondhand tobacco smoke, although differences in the concentrations of the components vary. ${ }^{34,35}$ Even in the absence of studies reporting the long-term health effects of passive exposure, clinicians should assess the risk of passive exposure in their patients and advise marijuana users to limit their use to open outdoor spaces where regulations permit, similar to tobacco use.

Using levels of cannabinoid or THC metabolites found in blood or urine samples to determine marijuana use or intoxication is challenging. There is no universal threshold that can differentiate between those who have actively smoked marijuana and are intoxicated, those who have actively smoked marijuana in the past and those who have been exposed to second-hand smoke. In many jurisdictions that have adopted thresholds for THC for drivers, $5.0 \mathrm{ng} / \mathrm{mL}$ for blood and $10 \mathrm{ng} / \mathrm{mL}$ for urine are common thresholds to indicate intoxication. ${ }^{36}$ In the studies included in this review, these levels were present 4-8 hours after exposure in those exposed to second-hand smoke. This raises questions about whether there should be tolerance for people who claim that their positive urine test result is due to second-hand exposure. ${ }^{37}$

As more jurisdictions legalize marijuana for recreational use, smokers may feel that use in common public areas or around children is acceptable, and, subsequently, harms associated with second-hand exposure may also increase. In the 
current state of the literature on second-hand exposure to marijuana smoke, it is difficult for clinicians to prepare to engage with patients in thorough assessments of marijuana exposure as they would with tobacco and for policy-makers to make evidence-based decisions. Future research to inform the development of effective communication tools, prevention strategies and policies to minimize harms to individual users and society is required.

Our systematic review did not identify any studies reporting the long-term effects of exposure to second-hand marijuana smoke or the effects of exposure to third-hand smoke. Participants were not followed beyond the experiment, and it is not known how repeated exposure to marijuana smoke may affect health. Given the known harms associated with active marijuana use, such as mental illness, brain developmental changes, respiratory and cardiac disease, and poor prenatal outcomes,,$^{2,38}$ the long-term impact of passive exposure requires further study. In the absence of evidence, based on the learnings from tobacco, a focus on harm reduction and limiting passive exposure may be prudent.

\section{Limitations}

One limit of our search strategy is that studies that were not in English or French were excluded, and the included studies were conducted primarily in anglophone countries. Furthermore, the included records are limited in transferability owing to small samples and the homogeneity of the population studied. The included studies were of good, fair or poor quality; no excellent studies were identified. The addition of excellentquality studies may have improved the robustness of our findings. The body of literature assessing exposure to secondhand marijuana smoke uses an experimental study design that may not be generalizable more broadly. However, it is likely that, under some regulatory conditions, people will be exposed in ways similar to those of the trials, which would enhance the generalizability of the findings to the real world. ${ }^{37}$ In addition, the included studies did not investigate effects in people who were repeatedly exposed to second-hand marijuana smoke, and all study participants were exposed for short periods. Exposure would likely be longer and more frequent if people were visiting a location where marijuana smoke was present, and, therefore, the generalizability of the results may be somewhat limited.

\section{Conclusion}

Tetrahydrocannabinol metabolites are retained in the body upward of 4 hours, and people report the experience of psychoactive effects after exposure to second-hand smoke. On a molecular level, marijuana smoke has chemical components similar to those of tobacco smoke, although they are present in different amounts. Although this provides support for the biological plausibility of the relation between exposure to secondhand marijuana smoke and negative health outcomes, there is a gap in the literature in this area. If exposure to second-hand marijuana smoke has similar health risks as direct marijuana use, it may be associated with conditions such as respiratory and cardiac disease as well as mental illness. However, high- quality research on the long- and short-term health effects of exposure to second-hand marijuana smoke are required to confirm these possible risks. Given the current state of knowledge, coherent policy approaches to exposure to smoke of any kind may result in the most effective harm-reduction policy.

\section{References}

1. Rotermann M, Langlois K. Prevalence and correlates of marijuana use in Canada, 2012. Health Rep 2015;26:10-5.

2. Volkow ND, Baler RD, Compton WM, et al. Adverse health effects of marijuana use. N Engl 7 Med 2014;370:2219-27.

3. National Cancer Institute, California Environmental Protection Agency. Health effects of exposure to environmental tobacco smoke: the report of the California Environmental Protection Agency. Smoking and Tobacco Control monographs, 10. NIH publication no. 99-4645. Bethesda (MD): US Department of Health and Human Services, Public Health Service, National Institutes of Health, National Cancer Institute; 1999.

4. Jinot J, Bayard S. Respiratory health effects of exposure to environmental tobacco smoke. Rev Environ Health 1996;11:89-100.

5. Simpson WJ. A preliminary report on cigarette smoking and the incidence of prematurity. Am f Obstet Gynecol 1957;73:807-15.

6. Aligne CA, Stoddard JJ. Tobacco and children: an economic evaluation of the medical effects of parental smoking. Arch Pediatr Adolesc Med 1997;151: 648-53.

7. Courage CM, Tamburlini G, von Ehrenstein OS. Environmental tobacco smoke. Geneva: World Health Organization; 2002.

8. Secondhand smoke [definition]. Merriam-Webster.com. Available: https:// www.merriam-webster.com/dictionary/secondhand\%20smoke (accessed 2016 Sept. 15).

9. Hecht SS. Carcinogen derived biomarkers: applications in studies of human exposure to secondhand tobacco smoke. Tob Control 2004;13(Suppl 1):i48-56.

10. Ferrante G, Simoni M, Cibella F, et al. Third-hand smoke exposure and health hazards in children. Monaldi Arch Chest Dis 2013;79:38-43.

11. Becquemin MH, Bertholon J, Bentayeb M, et al. Third-hand smoking: indoor measurements of concentration and sizes of cigarette smoke particles after resuspension. Tob Control 2010;19:347-8.

12. Rabin RC. A new cigarette hazard: 'third-hand smoke.' The New York Times 2009 Jan. 2.

13. Merritt TA, Mazela J, Adamczak A, et al. The impact of second-hand tobacco smoke exposure on pregnancy outcomes, infant health, and the threat of third-hand smoke exposure to our environment and to our children. Przegl Lek 2012;69:717-20.

14. Acuff L, Fristoe K, Hamblen J, et al. Third-hand smoke: old smoke, new concerns. 7 Community Health 2016;41:680-7.

15. Wang X, Derakhshandeh R, Liu J, et al. One minute of marijuana secondhand smoke exposure substantially impairs vascular endothelial function. 7 Am Heart Assoc 2016;5:e03858.

16. Moher D, Liberati A, Tetzlaff J, et al. Preferred reporting items for systematic reviews and meta-analyses: the PRISMA statement. PLoS Med 2009;6: e1000097.

17. Downs SH, Black N. The feasibility of creating a checklist for the assessment of the methodological quality both of randomised and non-randomised studies of health care interventions. 7 Epidemiol Community Health 1998;52: $377-84$

18. O'Connor SR, Tully MA, Ryan B, et al. Failure of a numerical quality assessment scale to identify potential risk of bias in a systematic review: a comparison study. BMC Res Notes 2015;8:224.

19. Cone EJ, Johnson RE, Darwin WD, et al. Passive inhalation of marijuana smoke: urinalysis and room air levels of delta-9-tetrahydrocannabinol. 7 Anal Toxicol 1987;11:89-96.

20. Niedbala RS, Kardos KW, Fritch DF, et al. Passive cannabis smoke exposure and oral fluid testing II. Two studies of extreme cannabis smoke exposure in a motor vehicle. 7 Anal Toxicol 2005;29:607-15.

21. Niedbala S, Kardos KW, Salamone S, et al. Passive cannabis smoke exposure and oral fluid testing. 7 Anal Toxicol 2004;28:546-52.

22. Cone EJ, Bigelow GE, Herrmann ES, et al. Nonsmoker exposure to secondhand cannabis smoke. III. Oral fluid and blood drug concentrations and corresponding subjective effects. 7 Anal Toxicol 2015;39:497-509.

23. Cone EJ, Bigelow GE, Herrmann ES, et al. Non-smoker exposure to secondhand cannabis smoke. I. Urine screening and confirmation results. 7 Anal Toxicol 2015;39:1-12.

24. Herrmann ES, Cone EJ, Mitchell JM, et al. Non-smoker exposure to secondhand cannabis smoke II: effect of room ventilation on the physiological, subjective, and behavioral/cognitive effects. Drug Alcohol Depend 2015;151:194-202.

25. Cone EJ, Roache JD, Johnson RE. Effects of passive exposure to marijuana smoke. Proceedings of the 48th Annual Scientific Meeting, the Committee on Problems of Drug Dependence, Inc. Taboe City, Nevada, Fune 1986. Rockville (MD): US Department of Health and Human Services; 1987. 


\section{Research}

26. Cone EJ, Roache JD, Johnson RE. Effects of passive exposure to marijuana smoke. NIDA Res Monogr 1987;76:150-6.

27. Law B, Mason PA, Moffat AC, et al. Passive inhalation of cannabis smoke. 7 Pharm Pharmacol 1984;36:578-81.

28. Moore C, Coulter C, Uges D, et al. Cannabinoids in oral fluid following passive exposure to marijuana smoke. Forensic Sci Int 2011;212:227-30.

29. Mulé S, Lomax P, Gross S. Active and realistic passive marijuana exposure tested by three immunoassays and GCIMS in urine. 7 Anal Toxicol 1988;12: 113-6.

30. Perez-Reyes M, Di Guiseppi S, Mason AP, et al. Passive inhalation of marihuana smoke and urinary excretion of cannabinoids. Clin Pharmacol Ther 1983;34:36-41.

31. Röhrich J, Schimmel I, Zörntlein S, et al. Concentrations of $\Delta 9$-tetrahydrocannabinol and 11-nor-9-carboxytetrahydrocannabinol in blood and urine after passive exposure to cannabis smoke in a coffee shop. 7 Anal Toxicol 2010; 34:196-203.

32. Zeidenberg P, Bourdon R, Nahas GG. Marijuana intoxication by passive inhalation: documentation by detection of urinary metabolites. Am 7 Psychiatry 1977;134:76-7.

33. Mørland J, Bugge A, Steen A, et al. Cannabinoids in blood and urine after passive inhalation of cannabis smoke. 7 Forensic Sci 1985;30:997-1002.

34. Maertens RM, White PA, Williams A, et al. A global toxicogenomic analysis investigating the mechanistic differences between tobacco and marijuana smoke condensates in vitro. Toxicology 2013;308:60-73.

35. Maertens RM, White PA, Rickert $W$, et al. The genotoxicity of mainstream and sidestream marijuana and tobacco smoke condensates. Chem Res Toxicol 2009;22:1406-14.
36. Wong K, Brady JE, Li G. Establishing legal limits for driving under the influence of marijuana. Inj Epidemiol 2014;1:26.

37. Hayden JW. Passive inhalation of marijuana smoke: a critical review. 7 Subst Abuse 1991;3:85-90.

38. Hall W, Degenhardt L. Adverse health effects of non-medical cannabis use. Lancet 2009;374:1383-91.

Affiliation: Department of Community Health Sciences and O'Brien Institute for Public Health, Cumming School of Medicine, University of Calgary, Calgary, Alta.

Contributors: Fiona Clement and Laura Dowsett conceived the study. Hannah Holitzki, Laura Dowsett and Fiona Clement designed the study, analyzed and interpreted the data and prepared the manuscript. Hannah Holitzki and Laura Dowsett collected and managed the data. All of the authors revised the manuscript for important intellectual content, gave final approval of the version to be published and agreed to be accountable for all aspects of the work.

Acknowledgement: The authors extend special thanks to Rebecca Saah of the University of Calgary for her help and critical comment on early drafts.

Supplemental information: For reviewer comments and the original submission of this manuscript, please see www.cmajopen.ca/content/5/4/ E814/suppl/DC1. 